\title{
A NOTE ON DUAL BANACH SPACES
}

\author{
STEN KAIJSER
}

\begin{abstract}
.
We prove a simple criterion for a Banach space to be a dual space, and we give some corollaries and applications to known dual spaces. Our theorem generalizes formally a theorem of Dixmier [3].
\end{abstract}

\section{Introduction.}

I have for a long time used "compactness of the unit ball" as a test of duality, believing it to be absolutely trivial to verify that a Banach space whose unit ball is compact for some linear Hausdorff topology is a dual space. I have, however, never seen this fact explicitly stated and when I finally decided to prove it, it turned out to be simple, but nevertheless the proof involves surprisingly deep theorems of functional analysis. Furthermore, I believe that at least the martingale version of the $H^{1}$ - BMO duality would probably have been discovered earlier if it had been recognized from the start that BMO is a dual space [4].

1.

We shall prove the following

THEOREM 1. Let $B$ be a Banach space, and let $E$ be a set of linear functions on B such that

(i) E separates points of $B$, and

(ii) the closed unit ball $U$ in $B$ is compact for the weak topology given by the set E.

Then $B$ is a dual space and the predual of $B$ is the closure in $B^{\prime}$ of the linear span of the set $E$.

Proof. Let $F$ be the norm closure in $B^{\prime}$ of the linear span of the set $E$. $F$ is then a closed linear subspace of $B^{\prime}$ so by the Hahn-Banach theorem the adjoint

Received March 18, 1977. 
$S^{\prime}$ of the embedding $S: F \rightarrow B^{\prime}$ maps the closed unit ball of $B^{\prime \prime}$ onto the unit ball of $F^{\prime}$. We shall prove that the restriction of $S^{\prime}$ to the unit ball $U$ of $B$ (considered as a subspace of $B^{\prime \prime}$ ) maps $U$ injectively onto the unit ball of $F^{\prime}$ and it follows then that $\left.S^{\prime}\right|_{B}$ maps $B$ isometrically onto $F^{\prime}$ so that $B$ is indeed a dual space. Let therefore $f^{\prime}$ be an element of the unit ball of $F^{\prime}$ and let $b^{\prime \prime}$ be a representative for $f^{\prime}$ in $B^{\prime \prime}$. Since $U$ is dense in the unit ball $U^{\prime \prime}$ of $B^{\prime \prime}$ for the weak topology with respect to $B^{\prime}$ there exists a net $\left\{b_{\alpha}\right\}_{\alpha \in A}$ in $U$ such that $\left\langle b_{\alpha}, b^{\prime}\right\rangle \rightarrow\left\langle b^{\prime \prime}, b^{\prime}\right\rangle$ for all $b^{\prime}$ in $B^{\prime}$. However, for the weak topology given by $E$ which coincides on $U$ with the weak topology given by $F, U$ is compact. There exists then a convergent subnet $\left\{b_{\beta}\right\}_{\beta \in B}$ converging to $b_{0} \in U$. But this simply means that for all $f$ in $F\left\langle b_{0}, f\right\rangle=\left\langle b^{\prime \prime}, f\right\rangle$ and this proves that $S^{\prime}$ maps $U$ onto the unit ball of $F^{\prime}$. Since the set $E$ separates points in $B$ the map $S^{\prime}$ is also injective and this proves the theorem.

REMARK. It follows from the assumptions that there is a separated duality between the Banach spaces $B$ and $F$, and by the construction of $F$ we also have

$$
\|f\|_{F}=\sup _{\|b\| \leqq 1}|\langle f, b\rangle| .
$$

Without the compactness assumption it is, however, not in general true that $\|b\|_{B}=\sup _{\|f\| \leqq 1}|\langle f, b\rangle|$. (Consider e.g. the duality between $B=c_{0}$ and $F$ the subspace of $l^{1}$ of all $\left\{a_{k}\right\}_{k=1}^{\infty}$ such that $\sum a_{k}=0$.)

Corollary 1. Let $K$ be a compact convex balanced subset of a locally convex vector space $E$ and let

$$
E_{K}=\{e \in E \mid \text { for some } t>0, t \cdot e \in K\} .
$$

$E_{K}$ is a Banach space with unit ball $K$ and this space is a dual space. The predual of $E_{K}$ is the completion of $E^{\prime}$ for the norm given by the duality with $E_{K}$.

SPECIAL CASE 1. If $K$ is a closed bounded convex and balanced subset of a reflexive space, then $E_{K}$ is a dual space.

SPECIAL CASE 2. If $E=V^{\prime}$ and $K=S^{0}$ where $S$ is a convex balanced neighborhood of 0 in $V$, then $E_{K}$ is a dual space. ( $S^{0}$ denoting the polar set in $E$.)

\section{Examples.}

We shall give some more or less well-known applications of our criterion.

a) Let $M, d$ be a metric space and let $\Lambda(M)$ be the Banach space of all functions $f$ on $M$ such that 
(i) $\|f\|_{\infty}=\sup _{m \in M}|f(m)|<\infty$

(ii) $\delta(f)=\sup _{m_{1} \neq m_{2}}\left(\left|f\left(m_{1}\right)-f\left(m_{2}\right)\right|\right) / d\left(m_{1}, m_{2}\right)<\infty$.

Then $\Lambda(M)$ is a dual space with the norm $\|f\|=\max \left(\|f\|_{\infty}, \delta(f)\right)$. The closed linear span in $\Lambda^{\prime}$ of the point masses on $M$ is the predual [1], [9], [8].

b) Let $M$ be a convex compact space, let $E$ be the space of all bounded functions on $M$ and let $K=P-P$, where $P$ is the set of all positive convex functions on $M$ that are bounded by 1 . Then $E_{K}$ is a dual space.

c) BMO is a dual space. The Banach space BMO was defined by John and Nirenberg [7]. There exist several definitions of BMO that are all equivalent but that give slightly different norms. Furthermore, BMO may be defined both for martingales and for functions on $\mathrm{R}^{n}[5]$, [6].

Since the space $B M O(R)$ is typical we only consider that space and to simplify the proof we define BMO $(R)$ as follows:

Let $f \in L_{\text {loc }}^{2}(\mathrm{R})$ (i.e. for every set $E$ of finite measure $f \in L^{2}(E)$ ), and let for every interval $I$

$$
E_{I}(f)=\frac{1}{|I|} \int_{I} f d x, \quad E_{I}^{2}(f)=E_{I}\left(\left|f-E_{I}(f)\right|^{2}\right) .
$$

Then $f \in \mathrm{BMO}$ if $\sup _{I}\left(E_{I}^{2}(f)\right)^{\frac{1}{2}}<\infty$.

Defining $\|f\|_{\mathrm{BMO}}=\sup _{I}\left(E_{I}^{2}(f)\right)^{\frac{1}{2}}$ it follows that a constant function has norm 0 . We consider therefore BMO as a subspace of $L_{\text {loc }}^{2} / \bmod$ constants. Letting $E$ be the reflexive space $L_{\text {loc }}^{2} / \bmod$ constants, and $K$ the unit ball of BMO it is immediate that $K$ is a bounded and closed subset of $E$, and therefore by special case $1, \mathrm{BMO}$ is a dual space. In order to obtain a representation of the predual we observe that for every interval $I$,

$$
\left(E_{I}^{2}(f)\right)^{\frac{1}{2}}=\sup \left\{E_{I}(f g) \mid g \in P_{I}\right\},
$$

where

$$
P_{I}=\left\{g \in L^{2}(I) \mid E_{I}(g)=0 \text { and } E_{I}\left(|g|^{2}\right) \leqq 1\right\} .
$$

But this means that

$$
\|f\|_{\text {Вмо }}=\sup \left\{\left|\int_{\mathrm{R}} f g d x\right|, g \in P\right\},
$$

where

$$
P=\left\{g \in L^{2}(\mathrm{R}) \mid g \text { has support in a compact interval } I,\right.
$$




$$
\left.\int g d x=0, \text { and }\|g\|_{L^{2}(I)} \leqq|I|^{\frac{1}{2}}\right\} .
$$

It follows that the elements in the predual of BMO have a representation

$$
h=\sum a_{i} g_{i}, \quad \sum\left|a_{i}\right|<\infty, \quad g_{i} \in P .
$$

We observe finally that $g \in P$ implies $\|g\|_{L^{1}(R)} \leqq 1$ so that the unit ball of the predual of BMO is contained in the unit ball of $L^{1}$ and all elements have integral 0 . Using the above approach the main difficulty in the proof of the Fefferman duality of $H^{1}$ and BMO is the identification of the predual constructed above with the space $H^{1}$ as given by some other definition.

Remark. Our representation of $H^{1}$ is what Coifman-Rochberg-Weiss call the representation in terms at atoms [2].

d) $H^{1}$ is a dual space. This lies deeper since it requires the F. and M. Riesz theorem. One defines thus $H^{1}$ as the set of all distributions that together with their Riesz transforms are measures.

\section{Classes of spaces.}

A) Various spaces of operators can by the same methods be proved to be dual spaces.

The trace-class operators on Hilbert-space is a dual space. Apply special case 1 of the corollary to the Hilbert-space $E$ of Hilbert-Schmidt operators, and let $K$ be the set of all $n$ in $E$ such that

$$
|\operatorname{Tr}(n e)| \leqq\|e\|_{\mathrm{op}} .
$$

(See [10].)

The fact that $E^{\prime} \hat{\otimes} F^{\prime}$ is a dual space whenever one of the spaces $E$ and $F$ is reflexive and one of $E^{\prime}$ and $F^{\prime}$ has the metric approximation property lies deeper and does not follow in a simple way from our general criteria.

On the other hand, such spaces as the set of all Hilbertian operators from $E$ to $F^{\prime}$, or all $p$-summing operators from $E$ to $F^{\prime}$ as well as the space of bounded quadratic forms, Hilbertian quadratic forms of $H^{\prime}$-quadratic forms on a Banach space are easily proved by these methods to be dual spaces.

B) A general class of spaces to which our theorems are easily applicable is the class of sequence spaces. Since the space $\Pi_{N} K$, i.e. the space of all sequences (of numbers from the field $K(=R$ or $=C$ )) is a reflexive space, it follows that if the unit ball of the sequence space $B$ is a closed bounded subset 
of $\prod_{N} K$ then $B$ is a dual space. Now the boundedness will usually be evident so the problem is to decide whether the unit ball is closed, and since it is certainly convex it is closed iff it is weakly closed. Now the dual of $\Pi K$ is $\amalg K$ i.e. the space of all sequences with finite support. This means that $B$ is a dual space if it can be determined in terms of linear functions with finite support. The guiding principle is that $B$ is a dual space unless the definition requires somewhere a $o(1)$ condition. (The sequence $\left\{x_{k}\right\}_{k=1}^{\infty}$ is $o(1)$ if $x_{k} \rightarrow 0$.)

C) For Banach spaces of Lebesgue-measurable functions things are slightly more complicated. First of all the "big space" $L_{\text {loc }}^{1}$ is not even a dual space. This implies that to use the corollary and in particular the special cases of the corollary the given space $B$ should preferably be contained at least in $L_{\text {loc }}^{p}$ for some $p>1$. However, if such a condition holds then the general principle is the same as for sequence spaces, i.e. $B$ is a dual space if the definition involves no $o(1)$. Alternatively, one may consider $B$ as a subspace of $M\left(\mathrm{R}^{d}\right)$ which is a dual space. The conditions on $B$ should imply uniform integrability and then the $o(1)$ principle usually applies.

D) We finally consider Banach spaces of continuous functions so let $X$ be a locally compact Hausdorff space and let $B$ be a Banach space such that $B \subset C(X)$. The main difficulty is of course that $C(X)$ is usually not a dual space - and if it is then the space $B$ is probably a sequence space or a space of measurable functions so that the space $C(X)$ is probably just another name for $l^{\infty}$ or $L^{\infty}$. To avoid this case we assume that $X$ is a metrizable space and if we wish to apply our corollary we let $B=E_{K}$ where $K$ is a compact subset of $E=C(X)$. The difficult problems arise when $E$ is given its weak topology so that $K$ is a weakly compact subset of $C(X)$. These problems are, however, of a much deeper nature than the problems considered in this note. We assume then that $K$ is compact for the norm topology in $C(X)$ and in that case the following proposition is worth mentioning.

Proposition 1. Let $X$ be a locally compact Hausdorff space, let $K$ be a convex balanced subset of $C(X)$ which is compact for the topology of uniform convergence on compact subsets and let $B=C(X)_{K}$. Then

(i) $B$ is a dual space and the inclusion $B \dot{C} C(X)$ is a compact map,

(ii) with respect to the metric $d(x, y)=\left\|\delta_{x}-\delta_{y}\right\|_{B^{\prime}}$ on $X$ one has $B \subset \Lambda(X, d)$ $\subset C(X)$.

Proof. (i) follows from Corollary 1 and the definition of compact maps while the first inclusion of (ii) follows from the definition of the metric $d$. It remains to prove that $\Lambda(X, d), \subset C(X)$ or equivalently that the topology on $X$ 
induced by the metric $d$ is weaker than the original topology, i.e. that the identity map of $X$ is continuous as a map $(X, \tau)$ ( $\tau$ the original topology) into $\left(X, \tau_{d}\right)\left(\tau_{d}\right.$ the topology induced by $\left.d\right)$. Writing now

$$
X \stackrel{\delta}{\longrightarrow} M(X) \stackrel{i^{\prime}}{\longrightarrow} B^{\prime}
$$

where $\delta$ is the map of $X$ into $M(X)$ given by $\delta(\mathrm{x})=\delta_{x}$ and $i^{\prime}$ the adjoint of $i$ : $B \subset C(X)$ we see that $\delta$ is continuous for the original topology on $X$ and the weak *-topology of $M(X)$ and $i^{\prime}$ is continuous for the weak *-topologies of $M(X)$ and $B^{\prime}$. Let now $x \in X$ and let $N$ be a compact neighborhood of $x$, then $\delta(N)$ is a compact subset of $M(X)$ and thus contained in a ball of radius $R$ say. But then $i^{\prime} \circ \delta(N)$ is contained in a norm-compact subset of $B^{\prime}$ and on normcompact subsets of $B^{\prime}$ the normtopology and the weak *-topology coincide. Therefore

$$
i^{\prime} \circ \delta: X \rightarrow B^{\prime}
$$

is continuous when $X$ is given its original topology and $B^{\prime}$ its norm topology, and this proves the proposition.

\section{REFERENCES}

1. R. Arens and J. Eells, Jr., On embedding uniform and topological spaces, Pacific J. Math. 6 (1956), 397-403.

2. R. R. Coifman, R. Rochberg and G. Weiss, Factorization theorems for Hardy spaces in several variables, Ann. of Math. 103 (1976), 611-635.

3. J. Dixmier, Sur un théorème de Banach, Duke Math. J. 15 (1948), 1057-1071.

4. C. L. Fefferman, Characterizations of bounded mean oscillation, Bull. Amer. Math. Soc. 77 (1971), 587-588.

5. C. L. Fefferman and E. M. Stein, $H^{p}$ spaces of several variables, Acta Math. 129 (1972), $137-$ 193.

6. A. M. Garsia, Martingale inequalities, W. A. Benjamin, Inc. Reading, Mass., 1973.

7. F. John and L. Nirenberg, On functions of bounded mean oscillation, Comm. Pure Appl. Math. 14 (1961), 785-799.

8. J. A. Johnson, Banach spaces of Lipschitz functions and vector-valued Lipschitz functions, Trans. Amer. Math. Soc. 148 (1970), 147-169.

9. L. V. Kantorovic and G. S. Rubinstein, On a space of completely additive functions, Vestnik Leningrad. Univ. 13 (1958), no. 7, 52-59 (Russian).

10. R. Schatten, $A$ theory of cross-spaces, Ann. of Math. Studies 26, Princeton Univ. Press, 1950.

MATEMATISKA INSTITUTIONEN

SYSSLOMANSGATAN 8

S-75223 UPPSALA

SWEDEN 